University of Nebraska - Lincoln

DigitalCommons@University of Nebraska - Lincoln

9-30-2002

\title{
Training of the exchange-bias effect in NiO-Fe heterostructures
}

\author{
A. Hochstrat \\ Laboratorium für Angewandte Physik, Gerhard-Mercator-Universität Duisburg, Duisburg, Germany \\ Christian Binek \\ University of Nebraska-Lincoln, cbinek@unl.edu \\ Wolfgang Kleemann \\ Laboratorium für Angewandte Physik, Gerhard-Mercator-Universität Duisburg, Duisburg, Germany, \\ wolfgang.kleemann@uni-due.de
}

Follow this and additional works at: https://digitalcommons.unl.edu/physicsbinek

Part of the Physics Commons

Hochstrat, A.; Binek, Christian; and Kleemann, Wolfgang, "Training of the exchange-bias effect in NiO-Fe heterostructures" (2002). Christian Binek Publications. 21.

https://digitalcommons.unl.edu/physicsbinek/21

This Article is brought to you for free and open access by the Research Papers in Physics and Astronomy at DigitalCommons@University of Nebraska - Lincoln. It has been accepted for inclusion in Christian Binek Publications by an authorized administrator of DigitalCommons@University of Nebraska - Lincoln. 


\title{
Training of the exchange-bias effect in NiO-Fe heterostructures
}

\author{
A. Hochstrat, Ch. Binek, and W. Kleemann \\ Laboratorium für Angewandte Physik, Gerhard-Mercator-Universität Duisburg, D-47048 Duisburg, Germany
}

(Received 28 May 2002; revised manuscript received 17 July 2002; published 30 September 2002)

\begin{abstract}
The training effect of a $\mathrm{NiO}(001) / \mathrm{Fe}(110)$ heterostructure is studied from magnetic hysteresis loops measured by superconducting quantum interference device magnetometry. Consecutive hysteresis loops exhibit a decreasing exchange bias effect. This behavior is known as the training effect, which reflects the dependence of the exchange bias field on the antiferromagnetic interface magnetization. In order to evidence this dependence, we study the decrease of the total saturation magnetization of the heterostructure for an increasing number of hysteresis cycles. Assuming proportionality between the interface magnetization and the total saturation magnetization, the description of the data is consistent within the phenomenological Meiklejohn Bean approach.
\end{abstract}

DOI: 10.1103/PhysRevB.66.092409

PACS number(s): 75.60.- d, 75.70.-i, 75.70.Cn

Ferromagnetic (FM) hysteresis and, in particular, hysteretic reversal of the magnetization in exchange bias (EB) heterostructures are both nonequilibrium phenomena from a thermodynamic point of view. The latter one describes a coupling phenomenon between the FM and an antiferromagnetic (AF) pinning layer, which gives rise to a shift of the FM hysteresis loop along the magnetic-field axis by an amount $H_{E B}$ the exchange bias field. EB in the case of compensated $\mathrm{AF}$ interfaces requires $\mathrm{AF}$ domains or other symmetrybreaking elements, which are able to create a net magnetization of the antiferromagnet, which gives rise to exchange coupling with the ferromagnet. Although mechanisms of domain formation like random fields ${ }^{1-3}$ or piezomagnetism, ${ }^{4-6}$ depend on the particular AF system, the AF domain state of a three-dimensional systems is, as a rule, metastable. This gives rise to nonequilibrium phenomena in the temperature or field driven change of magnetization. Hence it is not surprising that the EB shows a training effect as a consequence of metastability.

The training effect describes the decrease of the EB field when cycling the system through several consecutive hysteresis loops. Let $n$ be the number of such hysteresis loops. One often finds that the EB field after $n$ loops, $H_{E B}^{n}$, can be described by the proportionality $H_{E B}^{n}-H_{E B}^{\infty} \propto 1 / \sqrt{n}$. 7,8 The training effect in general has its origin in the reorientation of $\mathrm{AF}$ domains at the $\mathrm{AF} / \mathrm{FM}$ interface which takes place during each magnetization reversal of the FM top layer. ${ }^{3}$ As pointed out by Nogués and Schuller a pronounced training effect has been found in heterosystems involving polycrystalline $\mathrm{AF}$ pinning layers, ${ }^{9-11}$ while in single-crystalline pinning systems this effect is expected to be small. ${ }^{8}$ The grain size of a polycrystalline AF pinning substrate is an upper boundary for the correlation length of the AF order parameter. Hence polycrystallinity limits the long-range $\mathrm{AF}$ order and favors a metastable domain configuration. However, there are various other mechanisms, like structural disorder at the interface or impurity induced random fields, which give rise to $\mathrm{AF}$ domain formation and hence make a training effect possible in heterostructures involving singlecrystalline AF pinning layers. AF domain states have been extensively studied in the case of $\mathrm{NiO}^{12-18}$ Indeed, we ob- serve a distinct training effect when depositing a thin $\mathrm{Fe}$ layer on top of the compensated (001) surface of a $\mathrm{NiO}$ single crystal.

According to Meiklejohn and Bean ${ }^{19}$ the exchange bias is given by

$$
H_{E B}=-J \frac{S_{A F} S_{F M}}{\mu_{0} t_{F M} M_{F M}},
$$

where $J$ is the exchange integral between the $\mathrm{Ni}$ and $\mathrm{Fe}$ atoms, $S_{A F}$ and $S_{F M}$ are the interface magnetization of the antiferromagnet and of the ferromagnet respectively, $t_{F M}$ is the thickness of the Fe film and $M_{F M}$ is the spontaneous magnetization of the Fe layer. This simple but powerful formula suggests that the $\mathrm{AF}$ interface magnetization $S_{A F}$ is crucial for the EB, as previously shown in Refs. 20 and 21. In particular, the training effect is expected to originate from the decrease of $S_{A F}$ with increasing $n$, because all other quantities which enter Eq. (1) are expected to be independent of $n$. It is the aim of this paper, to evidence that the training effect originates from the $n$ dependence of $S_{A F}$. For this reason we precisely determine the saturation value of the magnetization of the total heterostructure and investigate its cycle dependence. The total saturation magnetization contains a constant as well as a cycle-dependent contribution. We show that the latter one exhibits the same $n$ dependence as the EB field and hence gives rise to the training effect.

A thin Fe layer of $12 \mathrm{~nm}$ thickness has been deposited under ultrahigh vacuum conditions on top of the compensated (001) surface of a thin $(0.1 \mathrm{~mm})$ single-crystalline $\mathrm{NiO}$ platelet. Its surface has been cleaned ex situ by ion-beam sputtering.

The NiO crystal was thermally stabilized at $T=142 \mathrm{~K}$ during Fe evaporation at a rate of $0.01 \mathrm{~nm} / \mathrm{s}$. This comparatively low growth temperature has been chosen in order to overcome the thermodynamically driven nonwetting behavior with the help of a kinetic growth mode which sets in at low temperatures. An additional double cap layer of 3.4-nm $\mathrm{Ag}$ and 50-nm Pt prevents oxidation of the Fe film and there 


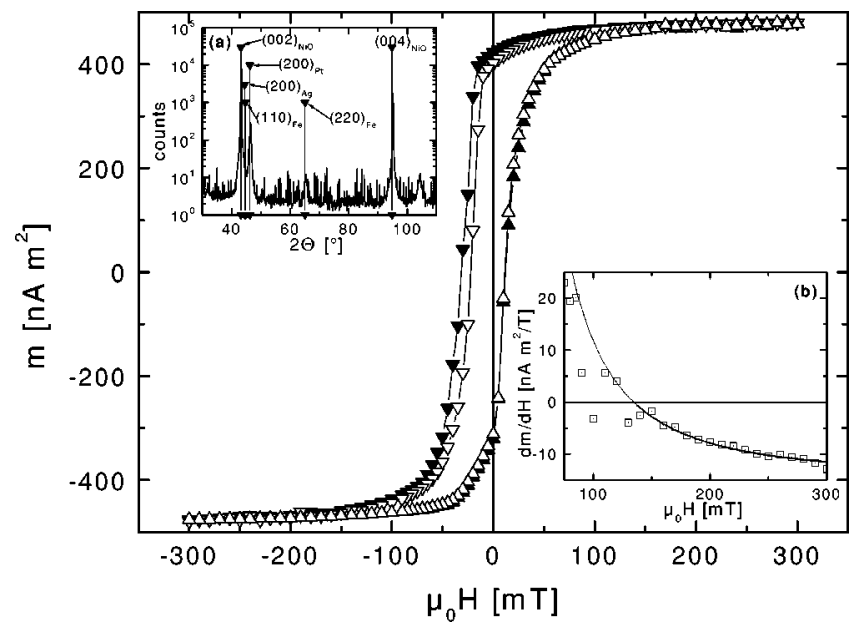

FIG. 1. Magnetic hysteresis curves of the $\mathrm{NiO} / \mathrm{Fe}$ heterostructure after subtraction of a linear background contribution. The solid and open triangles show the first and the ninth hysteresis loop of the virgin sample after field cooling, respectively. Up and down triangles indicate the up and down branch of the hysteresis, respectively. Inset (a) displays the x-ray-diffraction pattern with vertical lines indicating the Bragg peaks. Inset (b) shows the derivative $d m / d\left(\mu_{0} H\right)$ (dot centered squares) of the upper part of the down branch of one exemplary hysteresis curve. The bold solid line represents the fit (see text) to the corresponding data. The thin dotted line is an extrapolation of this fit to data outside of the fitting interval.

fore allows ex situ characterization. The inset (a) of Fig. 1 shows the result of large-angle $x$-ray diffraction using $\mathrm{Cu}_{K_{\alpha}}$ radiation. It exhibits (110) texturing of the $\mathrm{Fe}$ layer in accordance with the (110) and (220) Bragg peaks at $2 \Theta_{110_{F e}}=44.7^{\circ}$ and $2 \Theta_{220_{F e}}=65.1^{\circ}$, respectively. The (002) and (004) peaks at $2 \Theta_{002_{\mathrm{NiO}}}=43.2^{\circ}$ and $2 \Theta_{004_{\mathrm{NiO}}}$ $=94.8^{\circ}$ evidence the $(001)$ orientation of the surface of the $\mathrm{NiO}$ single crystal in accordance with previous $\mathrm{x}$-ray analyses. 22,23

After growing, the sample was cooled in a planar magnetic field of $0.5 \mathrm{~T}$ from $673 \mathrm{~K}$ down to room temperature under high vacuum condition. The freezing field possesses a positive sign with respect to the field axis defined in Fig. 1. This gives rise to the conventional negative exchange bias fields quantifying the shift of the loops which start close to positive saturation magnetization. This field-cooling procedure induces the unidirectional magnetic anisotropy, which gives rise to the EB. Starting from the virgin state of the heterostructure we subsequently measured magnetization hysteresis loops in a planar applied field at $T=5 \mathrm{~K}$ using a commercial superconducting quantum interference device (SQUID) magnetometer (Quantum Design: MPMS-5S).

The first loop exhibits an EB of $9.51 \mathrm{mT}$. It decreases with increasing $n$ down to $5.43 \mathrm{mT}$ for $n=9$. Figure 1 shows the first and ninth hysteresis loop (solid and open triangles, respectively) after subtraction of a small diamagnetic background contribution. The background subtraction turns out to be crucial for the determination of the absolute saturation values. Hence we describe the procedure in more detail. The total moment $m$ which is measured by SQUID magnetometry reads

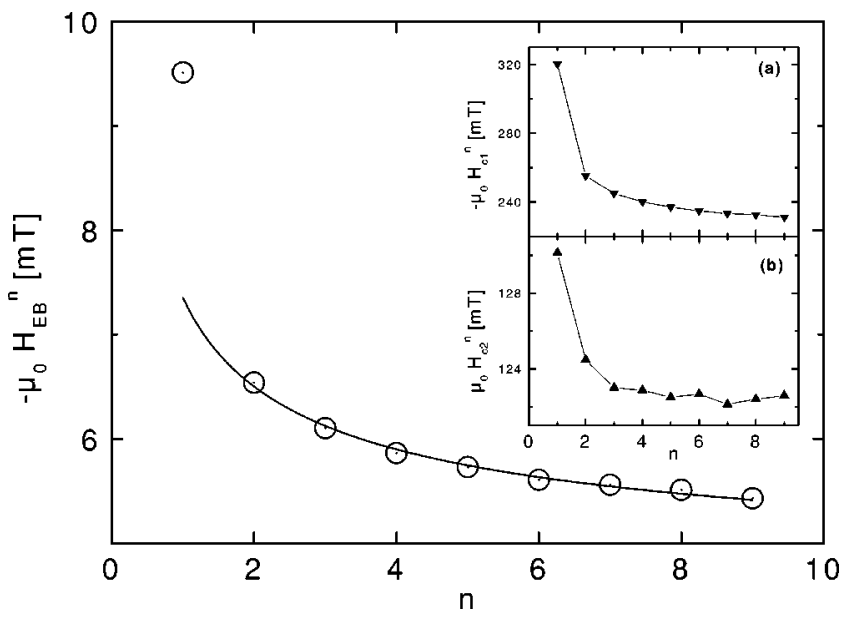

FIG. 2. Cycle-dependence of the exchange bias field (dot centered circles) and fit (solid line) of Eq. (4) to the data for $n>1$. Insets (a) and (b) show the cycle dependence of the coercive fields $H_{C_{1}}$ (down triangles) and $H_{C_{2}}$ (up triangles), respectively.

$$
m=m_{F M}+m_{A F}+m_{D},
$$

where $m_{F M}, m_{A F}$, and $m_{D}$ are the contributions of the FM Fe layer, the AF NiO single crystal, and a diamagnetic background contribution of the sample holder. The latter one is known to be an odd function of the magnetic field, which originates from a field-independent susceptibility, $\chi_{D}<0$. The $n$ dependence of $m$ originates from the AF contribution, $m_{A F}$. On the one hand, $m_{A F}$ contains the domain induced contribution which gives rise to the training effect. On the other hand, the AF susceptibility $\chi_{A F}>0$ gives rise to a fieldinduced, but reversible, component. Close to the saturation, where nucleation processes in the magnetization reversal of the Fe layer are negligible, the FM hysteresis can be described by the mean-field approach, ${ }^{24}$

$$
m_{F M}=m_{F M}^{0} L\left(\frac{g \mu_{B} \mu_{0}}{k_{B} T}\left(H+\lambda m_{F M}\right)\right),
$$

where $m_{F M}^{0}$ is the saturation moment and $L(x)$ is the Langevin function. In this field regime in good approximation $\lambda m_{F M}$ is constant with respect to the variation of $H$ which yields $\lambda m_{F M} \approx \lambda m_{F M}^{0}$. Using the derivative of Eq. (3) plus the additional constant $\chi=\chi_{D}+\chi_{A F}$, we fit the $d m / d\left(\mu_{0} H\right)$ vs $\mu_{0} H$ data of each of the nine hysteresis loops, respectively. As a result we obtain $\chi \approx-1.34 \times 10^{-8} \mathrm{Am}^{2} / \mathrm{T}$ from the arithmetic average of the nine particular results. A typical fit is shown in the inset of Fig. 1 (solid line). Only data close to the saturation where $d m / d\left(\mu_{0} H\right) \lesssim 0$ are involved in the fit. After subtraction of the background signal the EB field is calculated according to $H_{E B}=\frac{1}{2}\left(H_{C_{1}}\right.$ $+H_{C_{2}}$ ). The coercive fields $H_{C_{1}}$ and $H_{C_{2}}$ are determined from the intersections of the $m$ vs $H$ data with the field axis, where $H_{C_{1}}<H_{C_{2}}$ in accordance with the conventional definition. 
Figure 2 shows the EB field and the coercive fields [(a) and (b)] as functions of $n$. Obviously the training effect originates from the $n$ dependence of $H_{C_{1}}$ while $H_{C_{2}}$ shows a qualitatively similar but by far less pronounced dependence on $n$. The quantitatively different $n$ dependences of $H_{C_{1}}$ and $H_{C_{2}}$ indicate that the down and up branches of the hystereses follow different mechanisms of magnetization reversal. A similar behavior was found on $\mathrm{CoO} / \mathrm{Co}$ bilayers where coherent rotation has been observed at $H_{C_{2}}$ while domain nucleation and wall propagation dominates in the vicinity of $H_{C_{1}} \cdot{ }^{25-27}$ Moreover, in this case it was found that the first magnetization reversal in the virgin state is exceptional in the sense that pure $180^{\circ}$ domain-wall movement takes place at $H_{C_{1}}$. In accordance with these findings we observe in our $\mathrm{NiO} / \mathrm{Fe}$ heterostructure an exceptional large training effect between the first and the second hysteresis loop. As reported in very early work ${ }^{7}$ a similar behavior has been observed for various EB systems. The best fitted solid line in Fig. 2 shows that all subsequent data points $n \geqq 2$ of $-\mu_{0} H_{E B}$ vs $n$ follow nicely the proportionality

$$
H_{E B}^{n}-H_{E B}^{\infty} \propto \frac{1}{\sqrt{n}}
$$

The data point at $n=1$ significantly exceeds the value when extrapolating the fit to $n=1$ in accordance with previous investigations. ${ }^{7,8}$

In order to evidence the correlation between the training effect for $n>1$ and the decrease of $m_{A F}$ with increasing $n$ we calculate the total saturation magnetization of the heterostructure after subtraction of $\chi H$.

The smallness of the $n$ dependence of the total moment makes it necessary to take advantage from averaging the $m$ vs $H$ data of the $n$th down branch $m_{n}(H)$ according to

$$
\overline{m_{n}}=\frac{1}{\Delta H_{2}} \int_{H_{C 1}+\Delta H_{1}}^{H_{C_{1}}+\Delta H_{1}+\Delta H_{2}} m_{n}(H) d H
$$

where $\Delta H_{1}=149.5 \mathrm{mT}$ and $\Delta H_{2}=150.5 \mathrm{mT}$. Although $m_{A F}$ gives rise to a vertical shift of the hysteresis loop, which affects the positive as well as the negative part of a branch of a loop, we restrict our analysis to the positive part of the down branches, because the corresponding negative and positive parts of the up and down branches suffer from enhanced noise originating from flux creep that takes place in the superconducting coil of our magnetometer. Note that it is crucial to subtract the background before calculating $\overline{m_{n}}$, because the interval of integration shifts proportional with $H_{C_{1}}$. The shift of the interval of integration as a function of $H_{C_{1}}$ is of major importance in order to make sure that the integration takes place in the same quasisaturated FM state for each of the nine analyzed branches. Doing so, the above procedure prevents an artificial correlation between $\overline{m_{n}}$ and $H_{\mathrm{EB}}$.

The inset of Fig. 3 shows the $\overline{m_{n}}-\overline{m_{n=9}}$ vs $n$ data together with a best-fitted line according to expression (4). By

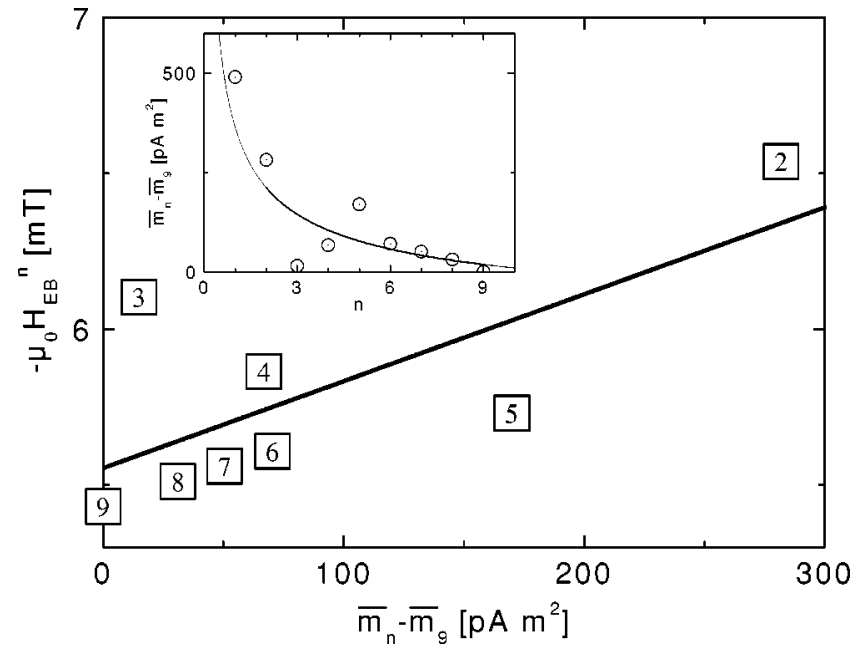

FIG. 3. Correlation between the exchange bias value $H_{E B}^{n}$ and the excess moment $\overline{m_{n}}-\overline{m_{9}}$ of the sample, where the small numbers inside the squares indicate $n$. The inset shows the cycle dependence of the excess moment (dot centered circles), a best-fitted line (solid line), and its extrapolation to cycle $n=1$, which is not involved in the fit.

this subtraction the large, but constant FM saturation magnetization $m_{F M} \approx 10^{3}\left(\overline{m_{1}}-\overline{m_{9}}\right)$ is eliminated. Obviously, the decrease of $\overline{m_{n}}-\overline{m_{9}}$ vs $n$ shows a qualitative similarity with the $n$ dependence of the EB field in Fig. 2. In order to evidence the simple correlation we plot $-\mu_{0} H_{E B}^{n}$ vs $\overline{m_{n}}-\overline{m_{9}}$ in Fig. 3. Note that the values $\overline{m_{1}}-\overline{m_{9}}$ come close to the limit of the SQUID sensitivity of approximately $100 \mathrm{pAm}^{2}$. Hence it is necessary to determine $\overline{m_{n}}$ from the statistical method outlined above. The straight line in Fig. 3 shows the result of a best linear fit. Within the accuracy of the measurement the data follow the linear behavior apart from the first point, which is known to reflect an extraordinary behavior. The data evidence that the training effect is correlated with a reduction of the AF moment. Unfortunately, an integral SQUID measurement cannot distinguish between the AF bulk and interface moment. However, under the assumption that $H_{E B}$ only depends on the $\mathrm{AF}$ interface magnetization a linear dependence of $H_{E B}$ on $S_{A F}$ can be deduced from the linearity between $H_{E B}$ and $\overline{m_{n}}-\overline{m_{9}}$.

It should be noticed that nonzero AF bulk magnetization $M_{A F}$ is expected to yield nonlinear contributions proportional to $M_{A F} S_{A F},{ }^{28}$ which are not confirmed by our present data within experimental accuracy. Instead the simple Meiklejohn Bean approach of Eq. (1) which points out a linear dependence of $H_{E B}^{n}$ on $S_{A F}$ is confirmed by our analysis of the training effect in $\mathrm{NiO} / \mathrm{Fe}$ heterostructure.

We measured the exchange bias and its training effect of a $\mathrm{NiO} / \mathrm{Fe}$ heterostructure by SQUID magnetometry. In accordance with recent results on $\mathrm{CoO} / \mathrm{Co},{ }^{27}$ the training effect originates mainly from the cycle-dependent shift for $H_{C_{1}}$ while $H_{C_{2}}$ remains virtually constant. The training effect is accompanied by a cycle-dependent decrease of the total satu- 
ration moment of the heterostructure. We evidence direct proportionality between the exchange bias and this total moment. The findings are in accordance with the phenomenological Meiklejohn Bean approach, where a linear dependence of the exchange bias on the AF interface magnetization is predicted.
We gratefully acknowledge support through the Sonderforschungsbereich 491 "Magnetische Heteroschichten: Struktur und elektronischer Transport" and the Graduiertenkolleg 277 "Struktur und Dynamik heterogener Systeme." Special thanks are due to W. Keune and his collaborators for discussions and technical support.
${ }^{1}$ W. Kleemann, Int. J. Mod. Phys. B 7, 2469 (1993).

${ }^{2}$ P. Miltenyi, M. Gierlings, J. Keller, B. Beschoten, G. Güntherodt, U. Nowak, and K.D. Usadel, Phys. Rev. Lett. 84, 4224 (2000).

${ }^{3}$ U. Nowak, A. Misra, and K.D. Usadel, J. Magn. Magn. Mater. 240, 243 (2002).

${ }^{4}$ Ch. Binek, Xi Chen, A. Hochstrat, and W. Kleemann, J. Magn. Magn. Mater. 240, 257 (2002).

${ }^{5}$ J. Kushauer, Ch. Binek, and W. Kleemann, J. Appl. Phys. 75, 5856 (1994).

${ }^{6}$ A.S. Borovik-Romanov, Zh. Eksp. Teor. Fiz. 38, 1088 (1960) [Sov. Phys. JETP 11, 786 (1960)].

${ }^{7}$ D. Paccard, C. Schlenker, O. Massenet, R. Montmory, and A. Yelon, Phys. Status Solidi 16, 301 (1966).

${ }^{8}$ J. Nogués and I.K. Schuller, J. Magn. Magn. Mater. 192, 203 (1999)

${ }^{9}$ C. Schlenker, S.S.P. Parkin, J.C. Scott, and K. Howard, J. Magn. Magn. Mater. 54, 801 (1986).

${ }^{10}$ K. Zhang, T. Zhao, and M. Fujiwara, J. Appl. Phys. 89, 6910 (2001)

${ }^{11}$ S.G. te Velthuis, A. Berger, and G.P. Felcher, J. Appl. Phys. 87, 5046 (2001).

${ }^{12}$ W.L. Roth, J. Appl. Phys. 31, 2000 (1960).

${ }^{13}$ W. Kleemann, F.J. Schäfer, and D.S. Tannhauser, J. Magn. Magn. Mater. 15, 415 (1980).

${ }^{14}$ H. Ohldag, A. Scholl, F. Nolting, S. Anders, F.U. Hillebrecht, and J. Stöhr, Phys. Rev. Lett. 86, 2878 (2001).

${ }^{15}$ F.U. Hillebrecht, H. Ohldag, N.B. Weber, C. Bethke, U. Mick, M. Weiss, and J. Bahrdt, Phys. Rev. Lett. 86, 3419 (2001).
${ }^{16}$ H. Ohldag, T.J. Regan, J. Stöhr, A. Scholl, F. Nolting, J. Lüning, C. Stamm, S. Anders, and R.L. White, Phys. Rev. Lett. 87, 247201 (2001).

${ }^{17}$ H. Matsuyama, C. Haginoya, and K. Koike, Phys. Rev. Lett. 85, 646 (2000).

${ }^{18}$ J.A. Borchers, Y. Ijiri, D.M. Lind, P.G. Ivanov, R.W. Erwin, A. Quasba, S.H. Lee, K.V. O'Donovan, and D.C. Dender, Appl. Phys. Lett. 77, 4187 (2000).

${ }^{19}$ W.H. Meiklejohn and C.P. Bean, Phys. Rev. 105, 904 (1957).

${ }^{20}$ Kentaro Takano, R.H. Kodama, A.E. Berkowitz, W. Cao, and G. Thomas, Phys. Rev. Lett. 79, 1130 (1997).

${ }^{21}$ J. Nogués, C. Leighton, and Ivan K. Schuller, Phys. Rev. B 61, 1315 (2000).

${ }^{22}$ R.P. Michel, A. Chaiken, C.T. Wang, and L.E. Johnson, Phys. Rev. B 58, 8566 (1998).

${ }^{23}$ H.P. Rooksby, Acta Crystallogr. 1, 226 (1948).

${ }^{24}$ S. Blundell, Magnetism in Condensed Matter (Oxford University Press, New York, 2001).

${ }^{25}$ V.I. Nikitenko, V.S. Gornakov, A.J. Shapiro, R.D. Shull, Kai Liu, S.M. Zhou, and C.L. Chien, Phys. Rev. Lett. 84, 765 (2000).

${ }^{26}$ M.R. Fitzsimmons, P. Yashar, C. Leighton, I.K. Schuller, J. Nogués, C.F. Majkrzak, and J.A. Dura, Phys. Rev. Lett. 84, 3986 (2000).

${ }^{27}$ F. Radu, M. Etzkorn, T. Schmitte, R. Siebrecht, A. Schreyer, K. Westerholt, and H. Zabel, J. Magn. Magn. Mater. 240, 251 (2002).

${ }^{28} \mathrm{Ch}$. Binek, A. Hochstrat, and W. Kleemann, J. Magn. Magn. Mater. 234, 353 (2001). 